\title{
SÔBRE A FECUNDIDADE E A DESOVA DA PESCADA-FOGUETE
}

(Recebido em 18/10/1963)

\author{
A. E. A. de M. Vazzoler
}

\section{INTRODUÇÃO}

Neste trabalho são apresentados os resultados obtidos no estudo da fecundidade e do tipo de desova da pescada-foguete ( $M a$ crodon ancylodon), espécie de grande representação dentro do total de pescado desembarcado em Santos (Richardson \& Moraes, 1960; Grupo de Pesquisas sôbre a Pesca Marítima, 1961).

O conhecimento da fecundidade é importante para o estudo quantitativo do estoque, ou seja, avaliação de seu tamanho e variações, e de seu potencial reprodutivo, o que permitiria previsões de produção futura.

\section{MATERIAL E MÉTODOS}

O material utilizado neste trabalho constou de 23 pescadas-foguetes capturadas no período de novembro de 1961 a março de 1962 , na costa meridional do Brasil, ao sul de $29^{\circ} \mathrm{S}$.

Coletou-se, do pescado desembarcado, 21 fêmeas com ovários em fase final de maturação e 2 fêmeas com ovários totalmente desovados. Dêsses peixes determinou-se o comprimento total $(\mathrm{cm})$ e o pêso total $(\mathrm{g})$, procurando-se obter fêmeas de tôdas as classes de comprimento. Os dois ovários, de cada peixe, foram retirados da cavidade abdominal; a seguir foram cortadas as membranas ováricas de ponta a ponta, e os ovários colocados em solução de Gilson, por um período de tempo suficiente para que, por agitação forte, se obtivesse separação completa dos óvulos, segundo o método de Tiews (1959). Êsse método nos permite tomar amostras ao acaso, do volume total de óvulos das duas gônadas.

Uma vez obtida a separação completa dos óvulos, o material foi lavado várias vêzes em álcool $70 \%$ para eliminar os resíduos

\footnotetext{
- Instituto Oceanográfico da Universidade de São Paulo. Membro do Grupo de Pesquisas sôbre a Pesca Maritima (G.P.P.M.).

Contr. no 26 do G.P.P.M.

Publ. no 187 do Inst. Ocean. da USP.
} 
dos tecidos. A massa total de óvulos das duas gônadas, dissociadas em álcool $70 \%$, foi colocada em uma proveta e foi lido o volume total da suspensão (óvulos + álcool 70\%); após 24 horas de sedimentação anotou-se o volume total dos óvulos.

A seguir determinou-se a precisão do método a ser utilizado na retirada de amostras para o cálculo do número total de óvulos. Escolheu-se como unidade de amostragem uma "Stempel-pipette" de 2 cc, agitando-se a suspensão antes de retirar as amostras, para se obter distribuição homogênea dos óvulos. Com as gônadas dissociadas, de um dos peixes, procedeu-se aos seguintes testes:

I - Tomou-se dois lotes de 10 amostras: no lote $A$ retirou-se as amostras sucessivamente, sem reposição e, no lote $B$ cada amostra foi reposta antes de ser retirada a seguinte. Contou-se o número de óvulos em cada amostra e testaram-se os resultados obtidos para os dois lotes.

O êrro padrão da média do lote $A(3,0 \%)$ e do lote $B(3,4 \%)$ indica ser suficiente a retirada e contagem dos óvulos de uma amostra de 2 cc.

Através do teste $t(\mathrm{t}=0,262 \therefore \mathrm{P}>5 \%)$ verificou-se não haver diferença significativa entre os dois lotes.

Assim sendo, as amostras foram retiradas segundo o método utilizado no lote $A$ e, para maior precisão, usou-se a média de duas amostras de 2 cc.

O número total de óvulos das duas gônadas (N) será dado pela expressão:

$$
\mathrm{N}=\frac{\mathrm{V} \cdot \mathrm{n}}{\mathrm{v}} \text {, onde: }
$$

$\mathrm{V}=$ volume total de óvulos das duas gônadas;

$\mathrm{n}$ = número médio de óvulos das duas amostras;

$\mathrm{v}=$ volume médio de óvulos das duas amostras.

II - Para testar se o valor assim obtido está próximo do real, calculou-se o número de óvulos das gônadas usadas como material de teste, usando-se a média dos valores obtidos para as duas primeiras amostras do lote $A$; a seguir, tomou-se $1 / 3$ do volume total de óvulos dessas gônadas e procedeu-se à contagem total, calculando-se depois o número de óvulos para o volume total.

Comparando-se os dois resultados, obteve-se uma diferença não significativa $(0,4 \%)$.

Com base nos resultados dos testes acima, consideramos os resultados obtidos através do método descrito como representativos do número real de óvulos existentes nas duas gônadas. 
Para se avaliar a fecundidade é necessário saber que parte do número total de óvulos existentes nas duas gônadas é eliminada por estação de desova. Para tanto, paralelamente à contagem, procedeu-se à medição do diâmetro dos óvulos, agrupando-se as medições em intervalos de 70 micra.

A distribuição de freqüência do diâmetro dos óvulos das gônadas totalmente desovadas mostrou que após a estação de desova restam nos ovários apenas ovócitos $(97,5 \%)$ com diâmetro inferior a 140 micra e um número muito reduzido de óvulos maiores $(2,5 \%)$, em reabsorção. Os ovócitos que permanecem nos ovários, após a desova, encontram-se representados na Figura 1 pela classe dos "menores de 140 micra".

Dêsse modo, para a avaliação da fecundidade foram considerados todos os óvulos com diâmetro superior a 140 micra.

A Figura 1 mostra as distribuições de freqüência dos diâmetros dos óvulos das gônadas em fase final de maturação, agrupados segundo a posição da última moda, procedimento empregado por Clark (1934), Howard \& Landa (1958), e outros autores, para demonstrar o desenvolvimento de grupos sucessivos de óvulos em maturação.

No Quadro I temos o número calculado de óvulos que seriam eliminados, na estação de desova 1961-1962, pelos 21 peixes examinados, bem como seu comprimento $(\mathrm{cm})$ e pêso $(\mathrm{g})$ totais.

\section{QUADRO I}

\begin{tabular}{rrrr}
\hline \multirow{2}{*}{.$^{\circ}$ do peixe } & Comprimento $(\mathrm{cm})$ & Pêso $(\mathrm{g})$ & Fecundidade \\
\hline \hline 1 & 26,5 & 155 & \\
2 & 27,5 & 180 & 26.210 \\
3 & 28,5 & 210 & 59.732 \\
4 & 29,0 & 220 & 42.737 \\
5 & 29,0 & 200 & 74.121 \\
6 & 29,5 & 210 & 90.070 \\
7 & 29,5 & 240 & 61.506 \\
8 & 31,5 & 300 & 127.454 \\
9 & 34,0 & 335 & 64.008 \\
10 & 34,0 & 400 & 80.120 \\
11 & 34,0 & 415 & 144.450 \\
12 & 34,0 & 400 & 132.875 \\
13 & 34,5 & 340 & 139.050 \\
14 & 34,5 & 330 & 83.551 \\
15 & 35,0 & 435 & 175.000 \\
16 & 35,0 & 435 & 149.364 \\
17 & 36,5 & 470 & 191.357 \\
18 & 36,5 & 465 & 118.858 \\
19 & 37,5 & 500 & 184.708 \\
20 & 37,5 & 485 & 217.632 \\
21 & 39,0 & 500 & 178.114 \\
\hline
\end{tabular}




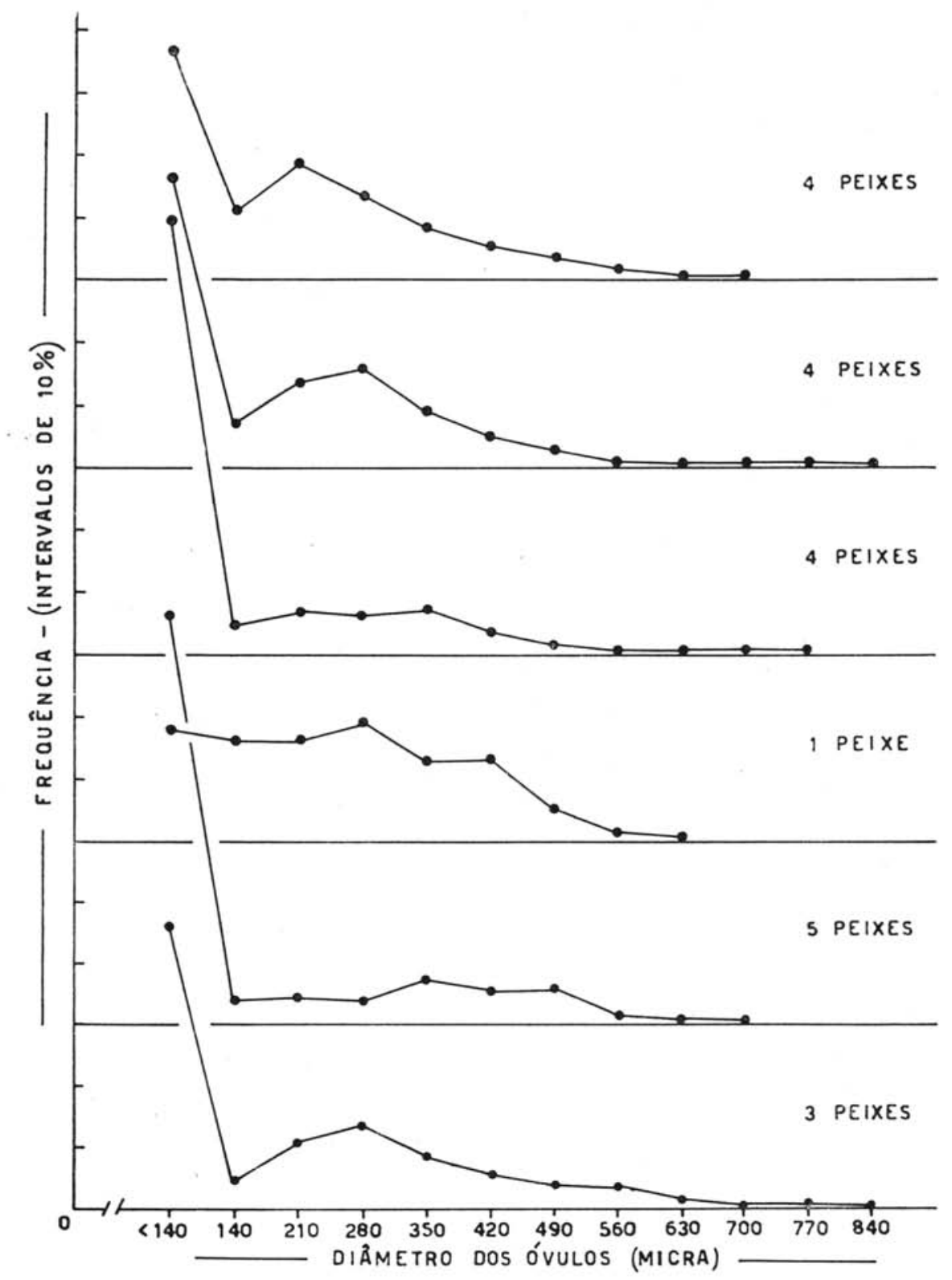

Fig. 1 - Distribuição da freqüência do diâmetro de óvulos de gônadas em fase final de maturação, agrupadas segundo a posição da última moda. 
Foram determinadas as correlações entre fecundidade e comprimento total (Fig. 2a) e pêso total (Fig. 2b).

\section{RESULTADOS E DISCUSSÃO}

A Figura 1 indica que os óvulos em maturação são eliminados em lotes sucessivos dentro da estação de desova; observa-se que, à medida que o desenvolvimento se processa, os óvulos maiores constituem uma moda, surgindo uma segunda, de óvulos menores, o que mostra que um segundo grupo de óvulos encontra-se em fase de crescimento, enquanto o primeiro já se encontra em maturação.

A ocorrência dêsse tipo de desova parcelada seria um dos fatôres que determinam a duração prolongada da época de reprodução, que se estende de novembro a maio (Yamaguti)*.

O cálculo da correlação (suposta linear) comprimento/fecundidade (Fig. 2a), através dos valores obtidos para cada classe de comprimento, foi feito pelo método dos mínimos quadrados, dando:

$$
\mathrm{F}=(12,7 \mathrm{~L}-299,5) 10^{3}
$$

( $\mathrm{r}=0,83 \therefore \mathrm{P}<1 \%$ ), onde $F=$ número de óvulos que serão eliminados, por fêmea, numa estação de desova e $L=$ comprimento total do peixe, em centímetros.

A considerável variação $\left(\sigma_{y}= \pm 32.120\right)$ encontrada para peixes de mesmo comprimento, provàvelmente, é devida ao fato dêles apresentarem pesos e idades diferentes (Simpson, 1951; Strzyzewska, 1962). Entretanto, nossos dados não são suficientes para determinarmos o efeito isolado de cada variável no valor da fecundidade.

Verificou-se que o comprimento para o qual $F=0(23,6 \mathrm{~cm})$ é próximo ao comprimento médio encontrado para o início da primeira maturação sexual $(26,0 \mathrm{~cm})$, vindo confirmar que o desenvolvimento sexual se processa num intervalo de classes de comprimento relativamente curto (Vazzoler, 1962).

O cálculo da correlação (suposta linear) pêso/fecundidade (Fig. 2b), através dos valores obtidos para cada pêso, foi feito pelo método dos mínimos quadrados, dando:

$$
\mathrm{F}=(40,5 \mathrm{~W}-186) 10^{2}
$$

( $\mathrm{r}=0,80 \therefore \mathrm{P}<1 \%$ ), onde $F=$ número de óvulos que serão eli-

- Yamaguti, N. - São Paulo, Instituto Oceanográfico. Membro do G.P.P.M., 1963. (Comunicação pessoal). 


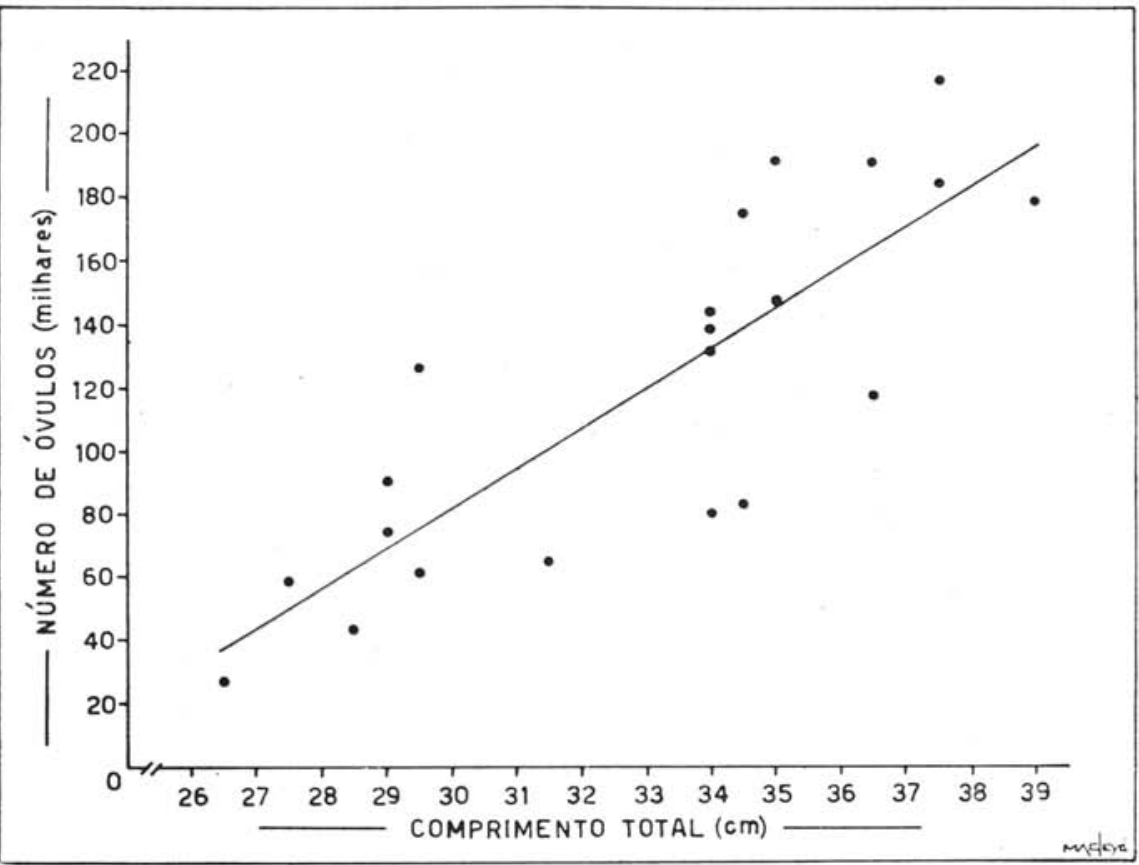

Fig. 2a - Representacão gráfica da relacão comprimento $(\mathrm{cm})$ e fecundidade (milhares de óvulos).

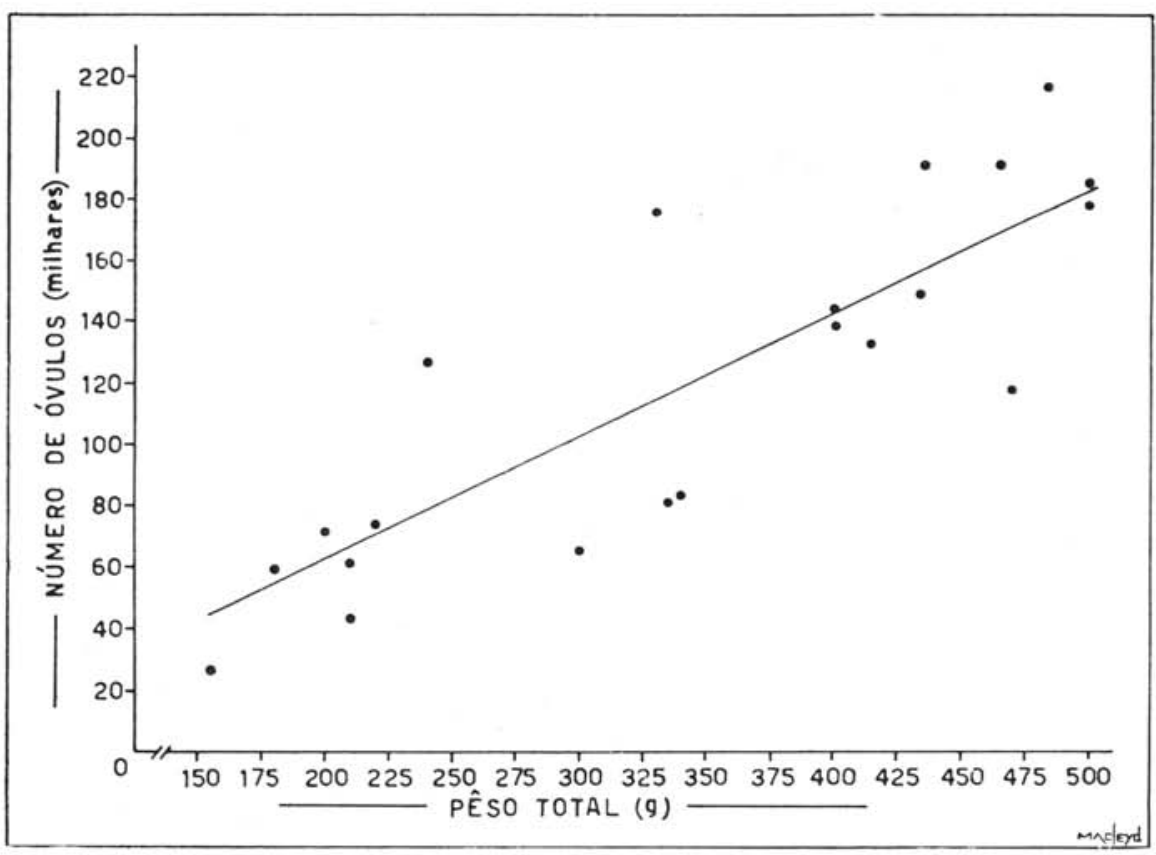

Fig. 2b - Representação gráfica da relação pêso total ( $g$ ) e fecundidade (milhares de óvulos). 
minados, por fêmea, em uma estação de desova e $W=$ pêso total do peixe, em gramas.

A variação encontrada $\left(\sigma_{y}= \pm 34.180\right)$ para peixes de mesmo pêso é ligeiramente maior que a encontrada para a correlação comprimento/fecundidade.

\section{CON CLUSÕ ES}

1 - A pescada-foguete apresenta distribuição do diâmetro dos óvulos polimodal, sendo que os óvulos são eliminados em lotes sucessivos, dentro da estação de desova.

2 - Êsse tipo de desova parcelada seria uma das causas do prolongado período de reprodução.

3 - Terminada a estação de desova, restam nos ovários apenas ovócitos $(97,5 \%)$ e uma pequena quantidade de óvulos maiores $(2,5 \%)$, em reabsorção.

4 - As correlações entre fecundidade e comprimento total $(\mathrm{cm})$ e pêso total $(\mathrm{g})$, mostram haver considerável variação da fecundidade em relação a cada parâmetro; entretanto, os dados não são suficientes para determinar-se o efeito isolado de cada um.

5 - Determinou-se, para $F=0$, o valor de $L$, sendo $23,6 \mathrm{~cm}$, valor próximo ao encontrado para o início da primeira maturação sexual.

\section{S U M M A R Y}

This paper presents an analysis of the fecundity and spawning of the sea trout, "pescada-foguete" (Macrodon ancylodon), of the Brazilian coast south of $29^{\circ} \mathrm{S}$, based mostly on egg count estimates of 21 specimens. The following conclusions were drawn:

1 - The eggs in the pre-spawning fish present a multimodal distribution of diameters, suggesting that they are spent successively within each spawning.

2 - The long spawning period, November-May, may be due to the partial spawning suggested above.

3 - In spent fish the ovaries contain mostly $(97.5 \%)$ ovocytes less than 140 micra in diameter, plus a small quantity $(2.5 \%)$ of eggs being resorbed.

4 - Correlations were drawn between fecundity $(F) /$ total length $(L)$ and fecundity $(F) /$ weight $(W)$, and are presented in equations (a) and (b) above,

5 - The value of $L$ for $F=0$ was determined and the value obtained $(23.6 \mathrm{~cm})$ is close to that verified for the beginning of the first maturity. 
B I B L I O G R A F I A

ClARK, F. N.

1934. Maturity of the California sardine (Sardina caerulea), determined by ova diameter measurements. Div. of Fish and Game of California, Fish Bull. n. ${ }^{\circ} 42$, p. 1-49.

Grupo de Pesquisas sôbre a Pesca Marítima, São Paulo.

1961. Tabelas de dados sôbre a pesca maritima: 1958-1959. Secr. Agr., Dep. Prod. Animal e Univ. de São Paulo, Inst. Ocean., 42 p.

Howard, G. V. \& LANDA, A.

1958. A study of the age, growth, sexual maturity, and spawning of the anchoveta (Cetengraulis mysticetus) in the Gulf of Panama. Inter-Amer. Trop. Tuna Comm., Bull. vol. II, n. ${ }^{\circ}$ 9, p. 391-467.

Richardson, I. D. \& Moraes, M. N.

1960. A first appraisal of the landing and mechanism of the Santos fishery. Bol. Inst. Ocean., vol. XI, n. ${ }^{\circ} 1$, p. 5-86.

Simpson, A. C.

1951. The fecundity of the plaice. Fish. Inv., Series II, vol. XVII, n. ${ }^{\circ} 5$, p. $1-27$.

STRZyZEWSKA, K.

1962. The changes in the fecundity of cod from the Gdansk Bay in the years 1959-1961. Intern. Counc. Expl. Sea, Gadoid Fish Committee, n. ${ }^{\circ} 119$, p. $1-2$.

TIEWS, K.

1959. Counting eggs for fecundity study. RTC/47, in: Lectures notes prepared for the International Training Centre on the Methodology and Techniques of Research on Macherel ( $R a$ strelliger), FAO, Rome, p. 1-2.

VAZzoler, A. E. A. DE M.

1962. Sôbre a primeira maturação sexual e destruição de peixes imaturos. Bol. Inst. Ocean., vol. XII, n. ${ }^{\circ}$ 2, p. 5-38. 